\title{
Discovery of TeV gamma-ray emission from the pulsar wind nebula 3C 58 by MAGIC
}

\author{
Rubén López-Coto ${ }^{a}$ for the MAGIC Collaboration \\ Institut de Física d'Altes Energies, Spain
}

\begin{abstract}
The pulsar wind nebula (PWN) 3C 58 is one of the historical very-high-energy (VHE; E $>100 \mathrm{GeV}$ ) gamma-ray source candidates. It has been compared to the Crab Nebula due to their morphological similarities. This object was detected by Fermi-LAT with a spectrum extending beyond $100 \mathrm{GeV}$. We analyzed 81 hours of 3C 58 data taken with the MAGIC telescopes and we detected VHE gamma-ray emission for the first time at $\mathrm{TeV}$ energies with a significance of 5.7 sigma and an integral flux of $0.65 \%$ C.U. above $1 \mathrm{TeV}$. According to our results 3C 58 is the least luminous PWN ever detected at VHE and the one with the lowest flux at VHE to date. We compare our results with the expectations of time-dependent models in which electrons up-scatter photon fields. The best representation favors a distance to the PWN of $2 \mathrm{kpc}$ and Far Infrared (FIR) comparable to CMB photon fields. Hadronic contribution from the hosting supernova remnant (SNR) requires unrealistic energy budget given the density of the medium, disfavoring cosmic ray acceleration in the SNR as origin of the VHE gammaray emission.
\end{abstract}

\section{General description}

The supernova remnant 3C 58 (SNR G130.7+3.1) has a flat radio spectrum and is brightest near the center, therefore it was classified as a pulsar wind nebula [PWN; 1]. It is centered on PSR J0205+6449, a pulsar discovered in 2002 with the Chandra X-ray observatory [2]. It is widely assumed that $3 \mathrm{C} 58$ is located at a distance of $3.2 \mathrm{kpc}$ [3], but recent $\mathrm{H}$ I measurements suggest a distance of $2 \mathrm{kpc}$ [4]. The age of the system is estimated to be $\sim 2.5 \mathrm{kyr}$ [5] from the PWN evolution and energetics, however this is a matter of debate. The pulsar has one of the highest spin-down powers known $\left(\dot{E}=2.7 \times 10^{37} \mathrm{erg} \mathrm{s}^{-1}\right)$. The PWN has a size of $9^{\prime} \times 6^{\prime}$ in radio, infrared (IR), and X-rays [6-9]. Its luminosity is $L_{0.5-10 \mathrm{keV}}=2.4 \times 10^{34} \mathrm{erg} \mathrm{s}^{-1}$ in the X-ray band, which is more than 3 orders of magnitude lower than that of the Crab nebula [10]. 3C 58 has been compared with the Crab because the jet-torus structure is similar [8]. Because of these morphological similarities with the Crab nebula and its high spin-down power (5\% of Crab), 3C 58 has historically been considered one of the PWNe most likely to emit $\gamma$ rays.

The PWN 3C 58 was previously observed in the VHE $\gamma$-ray range by several IACTs. The Whipple telescope reported an integral flux upper limit of $1.31 \times 10^{-11} \mathrm{~cm}^{-2} \mathrm{~s}^{-1} \sim 19 \%$ C.U. at an energy threshold of $500 \mathrm{GeV}$ [11], and VERITAS established upper limits at the level of $2.3 \%$ C.U. above an energy of $300 \mathrm{GeV}$ [12]. MAGIC-I observed the source in 2005 and established integral upper limits above $110 \mathrm{GeV}$ at the level of $7.7 \times 10^{-12} \mathrm{~cm}^{-2} \mathrm{~s}^{-1}(\sim 4 \%$

\footnotetext{
ae-mail: rlopez@ifae.es
}

(C) The Authors, published by EDP Sciences. This is an Open Access article distributed under the terms of the Creative Commons Attribution License 4.0 (http://creativecommons.org/licenses/by/4.0/). 


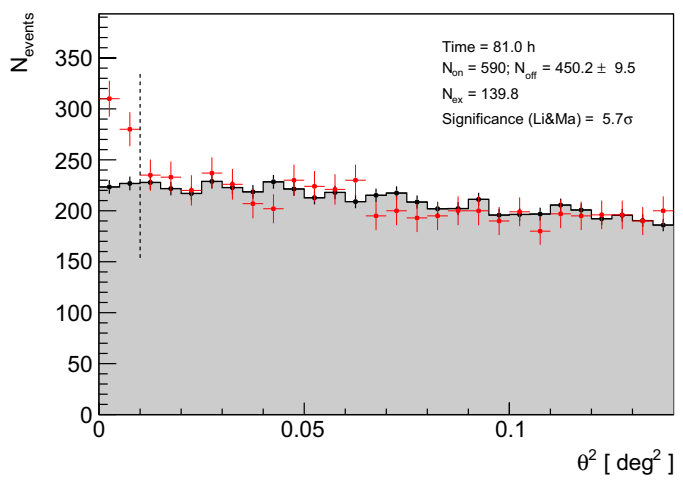

Figure 1. Distribution of squared angular distance, $\theta^{2}$, between the reconstructed arrival directions of gamma-ray candidate events and the position of PSR J0205+6449 (red points).

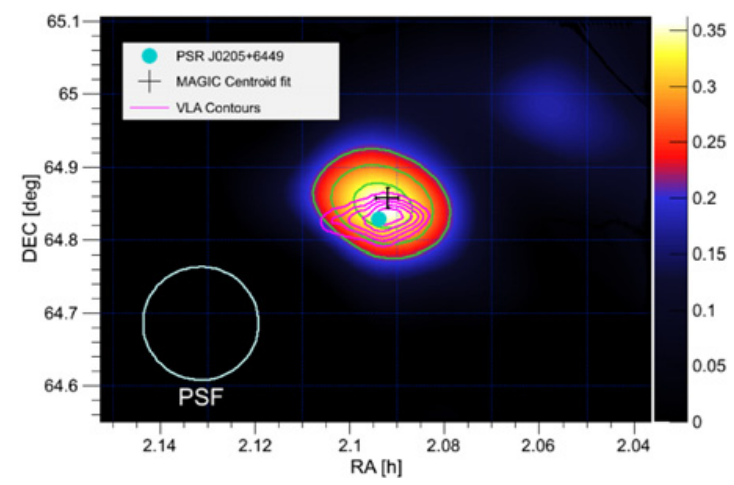

Figure 2. Relative flux (excess/background) map for MAGIC observations. The cyan circle indicates the position of PSR J0205+6449 and the black cross shows the fitted centroid of the MAGIC image with its statistical uncertainty. In green we plot the contour levels for the TS starting at 4 and increasing in steps of 1 . The magenta contours represent the VLA flux at $1.4 \mathrm{GHz}$ [16], starting at $0.25 \mathrm{Jy}$ and increasing in steps of $0.25 \mathrm{Jy}$.

C.U.) $[13,14]$. The improved sensitivity of the MAGIC telescopes with respect to previous observations and the Fermi-LAT results motivated us to perform deep VHE observations of the source.

\section{MAGIC observations and results}

MAGIC observed 3C 58 in the period between 4 August 2013 to 5 January 2014 for 99 hours, and after quality cuts, 81 hours of the data were used for the analysis. The data were analyzed using the MARS analysis framework [15].

The applied cuts yield an energy threshold of $420 \mathrm{GeV}$. The significance of the signal, calculated with the LiMa formula, is $5.7 \sigma$, which establishes 3 C 58 as a $\gamma$-ray source. The $\theta^{2}$ distribution is shown in Fig. 1. As the five OFF positions were taken for each of the wobble positions, the OFF histograms were re-weighted depending on the time taken on each wobble position.

We show in Fig. 2 the relative flux (excess/background) skymap, produced using the same cuts as for the $\theta^{2}$ calculation. The TS significance, which is the LiMa significance applied on 


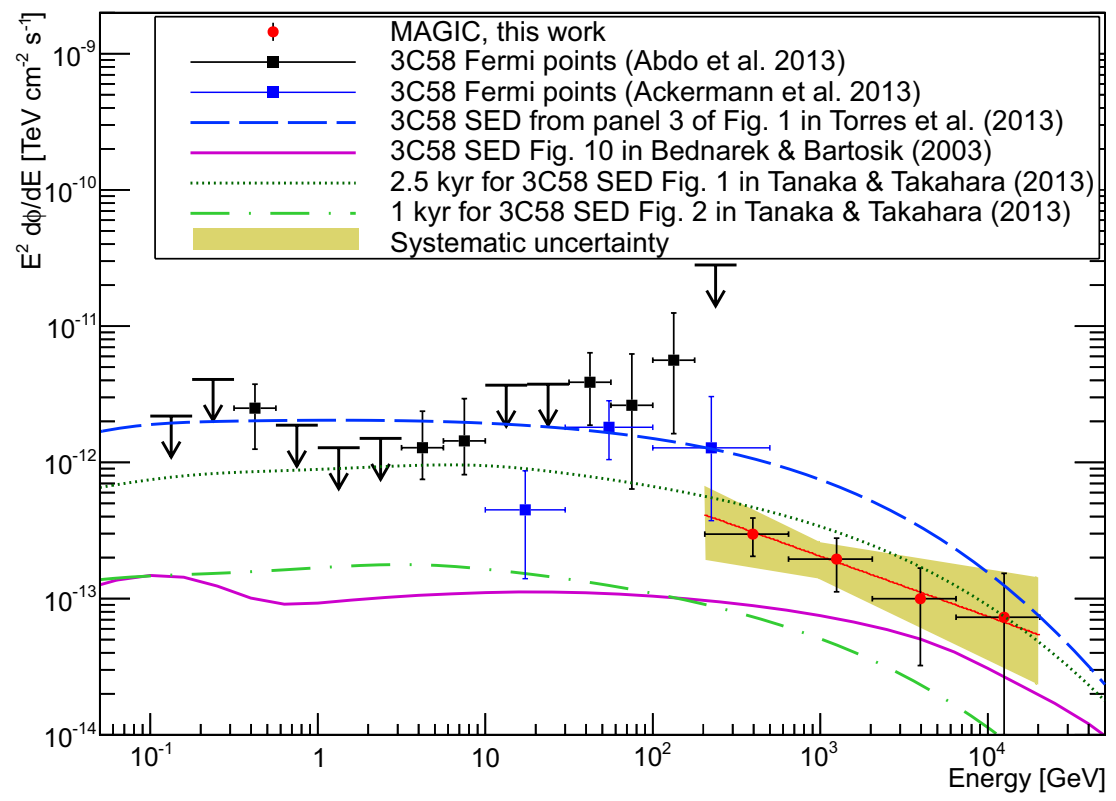

Figure 3. 3C 58 spectral energy distribution in the range between $0.1 \mathrm{GeV}$ and $20 \mathrm{TeV}$. Red circles are the VHE points reported in this work. The best-fit function is drawn in red and the systematic uncertainty is represented by the yellow shaded area. Black squares and black arrows are taken from the Fermi-LAT second pulsar-catalog results [17]. Blue squares are taken from the Fermi high-energy LAT catalog [18]. The magenta line is the SED prediction for 3C 58 taken from Fig. 10 of [21]. The clear green dashed-dotted line is the SED predicted by [22], assuming an age of $1 \mathrm{kyr}$, and the dark green dotted line is the prediction from the same paper, assuming an age of $2.5 \mathrm{kyr}$. The blue dashed line represents the SED predicted by [23] assuming that the Galactic FIR background is high enough to reach a flux detectable by the MAGIC sensitivity in $50 \mathrm{~h}$.

a smoothed and modeled background estimate, is higher than 6 at the position of the pulsar PSR J0205+6449. The excess of the VHE skymap was fit with a Gaussian function. The best-fit position is $\operatorname{RA}(\mathrm{J} 2000)=2 \mathrm{~h} 05 \mathrm{~m} 31(09)_{\text {stat }}(11)_{\text {sys }} \mathrm{s}$; DEC $(\mathrm{J} 2000)=64^{\circ} 51^{\prime}(1)_{\text {stat }}(1)_{\text {sys }}$. This position is statistically deviant by $2 \sigma$ from the position of the pulsar, but is compatible with it if systematic errors are taken into account. In the bottom left of the image we show the point spread function (PSF) of the smeared map at the corresponding energies, which is the result of the sum in quadrature of the instrumental angular resolution and the applied smearing ( $4.7^{\prime}$ radius, at the analysis energy threshold). The extension of the signal is compatible with the instrument PSF. The VLA contours are coincident with the detected $\gamma$-ray excess.

Figure 3 shows the energy spectrum for the MAGIC data, together with published predictions for the gamma-ray emission from several authors, and two spectra obtained with three years of Fermi-LAT data, which were retrieved from the Fermi-LAT second pulsar-catalog [2PC, 17] and the Fermi high-energy LAT catalog [1FHL, 18]. The 1FHL catalog used events from the Pass 7 Clean class, which provides a substantial reduction of residual cosmic-ray background above $10 \mathrm{GeV}$, at the expense of a slightly smaller collection area, compared with the Pass 7 Source class that was adopted for 2PC [19]. The two $\gamma$ ray spectra from $3 \mathrm{C} 58$ reported in the $2 \mathrm{PC}$ and $1 \mathrm{FHL}$ catalogs agree within statistical uncertainties. The differential energy spectrum of the source is well fit by a single powerlaw function $\mathrm{d} \phi / \mathrm{d} E=f_{0}(E / 1 \mathrm{TeV})^{-\Gamma}$ with $f_{0}=\left(2.0 \pm 0.4_{\text {stat }} \pm 0.6_{\text {sys }}\right) 10^{-13} \mathrm{~cm}^{-2} \mathrm{~s}^{-1} \mathrm{TeV}^{-1}$, 
$\Gamma=2.4 \pm 0.2_{\text {stat }} \pm 0.2_{\text {sys }}$ and $\chi^{2}=0.04 / 2$. The systematic errors were estimated from the MAGIC performance paper [20] including the upgraded telescope performances. The integral flux above $1 \mathrm{TeV}$ is $F_{E>1 \mathrm{TeV}}=1.4 \times 10^{-13} \mathrm{~cm}^{-2} \mathrm{~s}^{-1}$. Taking into account a distance of $2 \mathrm{kpc}$, the luminosity of the source above $1 \mathrm{TeV}$ is $L_{\gamma}, E>1 \mathrm{TeV}=(3.0 \pm 1.1) \times 10^{32} \mathrm{~d}_{2}^{2} \mathrm{erg} \mathrm{s}^{-1}$, where $d_{2}$ is the distance normalized to $2 \mathrm{kpc}$.

\section{Discussion}

Several models have been proposed that predict the VHE $\gamma$-ray emission of PWN 3C 58. [24] presented a one zone model of the spectral evolution of PWNe and applied it to 3C 58 using a distance of $3.2 \mathrm{kpc}$. The VHE emission from this model consists of IC scattering of CMB photons and optical-to-IR photons, and also of pion decay. The flux of $\gamma$ rays above $400 \mathrm{GeV}$ predicted by this model is about an order of magnitude lower than the observation.

[21] proposed a time-dependent model in which positrons gain energy in the process of resonant scattering by heavy nuclei. The VHE emission is produced by IC scattering of leptons off $\mathrm{CMB}$, IR, and synchrotron photons and by the decay of pions due to the interaction of nuclei with the matter of the nebula. The age of $3 \mathrm{C} 58$ is assumed to be $5 \mathrm{kyr}$, using a distance of $3.2 \mathrm{kpc}$ and an expansion velocity of $1000 \mathrm{~km} \mathrm{~s}^{-1}$. According to this model, the predicted integral flux above $400 \mathrm{GeV}$ is $\sim 10^{-13} \mathrm{~cm}^{-2} \mathrm{~s}^{-1}$, while the integral flux above $420 \mathrm{GeV}$ measured here is $5 \times 10^{-13} \mathrm{~cm}^{-2} \mathrm{~s}^{-1}$. Calculations by [25], using the same model with an initial expansion velocity of $2000 \mathrm{~km} \mathrm{~s}^{-1}$ and considering IC scattering only from the $\mathrm{CMB}$, are consistent with the observed spectrum. However, the magnetic field derived in this case is $B \sim 14 \mu \mathrm{G}$ and it underestimates the radio emission of the nebula, although a more complex spectral shape might account for the radio nebula emission.

[26] developed a time-dependent model of the spectral evolution of PWN including synchrotron emission, synchrotron self-Compton, and IC. They evolved the electron energy distribution using an advective differential equation. To calculate the observability of $3 \mathrm{C} 58$ at $\mathrm{TeV}$ energies they assumed a distance of $2 \mathrm{kpc}$ and two different ages: $2.5 \mathrm{kyr}$ and $1 \mathrm{kyr}$ [22]. For the $2.5 \mathrm{kyr}$ age, they obtained a magnetic field $B \sim 17 \mu \mathrm{G}$, while for an age of $1 \mathrm{kyr}$, the magnetic field obtained is $\mathrm{B}=40 \mu \mathrm{G}$. The emission predicted by this model is closer to the Fermi result for an age of $2.5 \mathrm{kyr}$.

[27] presented a different time-dependent leptonic diffusion-loss equation model without approximations, including synchrotron emission, synchrotron self-Compton, IC, and bremsstrahlung. They assumed a distance of $3.2 \mathrm{kpc}$ and an age of $2.5 \mathrm{kyr}$ to calculate the observability of 3C 58 at high energies [23]. The predicted emission, without considering any additional photon source other than the $\mathrm{CMB}$, is more than an order of magnitude lower than the flux reported here. It predicts VHE emission detectable by MAGIC in 50 hours for an FIR-dominated photon background with an energy density of $5 \mathrm{eV} / \mathrm{cm}^{3}$. This would be more than one order of magnitude higher than the local IR density in the Galactic background radiation model used in GALPROP $\left[\sim 0.2 \mathrm{eV} \mathrm{cm}^{-3} ; 28\right]$. The magnetic field derived from this model is $35 \mu \mathrm{G}$. To reproduce the observations, a large FIR background or a revised distance to the PWN of $2 \mathrm{kpc}$ are required. In the first case, a nearby star or the SNR itself might provide the necessary FIR targets, although no detection of an enhancement has been found in the direction of the PWN. As we mentioned in Sect. 1, a distance of $2 \mathrm{kpc}$ has recently been proposed by [4] based on the recent H I measurements of the Canadian Galactic Plane Survey. At this distance, a lower photon density is required to fit the VHE data. 


\section{Conclusions}

We have for the first time detected VHE $\gamma$ rays up to TeV energies from the PWN 3C 58. Following the assumptions in [29], it is highly unlikely that the measured flux comes from hadronic emission of the SNR. The measured luminosity and flux make 3C 58 into an exceptional object. It is the weakest VHE PWN detected to date, a fact that attests to the sensitivity of MAGIC. On the other hand, it is also the least luminous VHE PWN, far less luminous than the original expectations. Its ration $L_{V H E} / \dot{E} \simeq 10^{-5}$ is the lowest measured, similar to Crab, which makes into a very inefficient $\gamma$-ray emitter. Only a closer distance of $2 \mathrm{kpc}$ or a high local FIR photon density can qualitatively reproduce the multiwavelength data of this object in the published models. Since the high FIR density is unexpected, the closer distance with FIR photon density comparable with the averaged value in the Galaxy is favored. The models that fit the $\gamma$-ray data derived magnetic fields which are very far from equipartition.

\section{References}

[1] K.W. Weiler, N. Panagia, A\&A 70, 419 (1978)

[2] S.S. Murray, P.O. Slane, F.D. Seward, S.M. Ransom, B.M. Gaensler, ApJ 568, 226 (2002), astro-ph/0108489

[3] D.A. Roberts, W.M. Goss, P.M.W. Kalberla, U. Herbstmeier, U.J. Schwarz, A\&A 274, 427 (1993)

[4] R. Kothes, A\&A 560, A18 (2013)

[5] R.A. Chevalier, ApJ 619, 839 (2005), arXiv:astro-ph/0409013

[6] M.F. Bietenholz, N.E. Kassim, K.W. Weiler, ApJ 560, 772 (2001), arXiv:astro$\mathrm{ph} / 0106334$

[7] F. Bocchino, R.S. Warwick, P. Marty, D. Lumb, W. Becker, C. Pigot, A\&A 369, 1078 (2001), astro-ph/0102087

[8] P. Slane, D.J. Helfand, E. van der Swaluw, S.S. Murray, ApJ 616, 403 (2004), arXiv:astro-ph/0405380

[9] P. Slane, D.J. Helfand, S.P. Reynolds, B.M. Gaensler, A. Lemiere, Z. Wang, ApJ 676, L33 (2008), 0802.0206

[10] K. Torii, P.O. Slane, K. Kinugasa, K. Hashimotodani, H. Tsunemi PASJ 52, 875 (2000), astro-ph/0006034

[11] T.A. Hall, S.P. Wakely, VERITAS Collaboration, Observations of Galactic Pulsars and Shell-Type SNRs with the Whipple 10 m Imaging Atmospheric Cherenkov Telescope, in International Cosmic Ray Conference (2001), Vol. 6 of International Cosmic Ray Conference, p. 2485

[12] E. Aliu, Search for VHE y-ray emission in the vicinity of selected pulsars of the Northern Sky with VERITAS, in American Institute of Physics Conference Series, edited by F.A. Aharonian, W. Hofmann, F. Rieger (2008), Vol. 1085 of American Institute of Physics Conference Series, pp. 324-327, 0812.1415

[13] H. Anderhub, L.A. Antonelli, P. Antoranz, M. Backes, C. Baixeras, S. Balestra, J.A. Barrio, D. Bastieri, J. Becerra González, J.K. Becker et al., ApJ 710, 828 (2010)

[14] E. Aliu (2007), vHE gamma-ray observations of Northern sky pulsar wind nebulae with the MAGIC telescope, $\mathrm{PhD}$. thesis

[15] R. Zanin, E. Carmona, J. Sitarek, P. Colin, K. Frantzen, M. Gaug, S. Lombardi, for the MAGIC collaboration, Proceedings of the ICRC 2013, id 773 (2013), 0907.0943

[16] J.J. Condon, W.D. Cotton, E.W. Greisen, Q.F. Yin, R.A. Perley, G.B. Taylor, J.J. Broderick, AJ 115, 1693 (1998) 
[17] A.A. Abdo, M. Ajello, A. Allafort, L. Baldini, J. Ballet, G. Barbiellini, M.G. Baring, D. Bastieri, A. Belfiore, R. Bellazzini et al., ApJ 208, 17 (2013), 1305. 4385

[18] The Fermi-LAT Collaboration, ArXiv e-prints (2013), 1306.6772

[19] M. Ackermann, M. Ajello, A. Albert, A. Allafort, W.B. Atwood, M. Axelsson, L. Baldini, J. Ballet, G. Barbiellini, D. Bastieri et al., ApJ 203, 4 (2012), 1206.1896

[20] J. Aleksić, E.A. Alvarez, L.A. Antonelli, P. Antoranz, M. Asensio, M. Backes, J.A. Barrio, D. Bastieri, J. Becerra González, W. Bednarek et al., Astroparticle Physics 35, 435 (2012), 1108.1477

[21] W. Bednarek, M. Bartosik, A\&A 405, 689 (2003), arXiv:astro-ph/0304049

[22] S.J. Tanaka, F. Takahara, MNRAS 429, 2945 (2013), 1211.7266

[23] D.F. Torres, A.N. Cillis, J. Martín Rodriguez, ApJ 763, L4 (2013), 1212. 1995

[24] N. Bucciantini, J. Arons, E. Amato, MNRAS 410, 381 (2011)

[25] W. Bednarek, M. Bartosik, Journal of Physics G Nuclear Physics 31, 1465 (2005), arXiv: astro-ph/0509872

[26] S.J. Tanaka, F. Takahara, ApJ 715, 1248 (2010), 1004.3098

[27] J. Martín, D.F. Torres, N. Rea, MNRAS 427, 415 (2012), 1209. 0300

[28] T.A. Porter, I.V. Moskalenko, A.W. Strong, ApJ 648, L29 (2006), astro-ph/0607344

[29] L.O. Drury, F.A. Aharonian, H.J. Voelk, A\&A 287, 959 (1994), astro-ph/9305037 